\title{
Lecture
}

\section{Cryptography and Society ${ }^{\#}$}

\author{
R BALASUBRAMANIAN $^{1}$ and M PREM LAXMAN DAS ${ }^{2}$ \\ ${ }^{1}$ The Institute of Mathematical Sciences (IMSc), CIT Campus, Taramani, Chennai 600 113, Tamil Nadu, \\ India \\ ${ }^{2}$ Society for Electronic Transactions and Security (SETS), MGR Knowledge City, CIT Campus, Taramani, \\ Chennai 600 113, Tamil Nadu, India
}

(Delivered on 17 December 2019)

\begin{abstract}
From banking transactions to communicating with our near and dear, many operations are being carried out over the net nowadays. The importance of securing every such communication was well recognized. From being a niche topic researched by defence and government establishments, cryptography has become a subject studied by computer scientists, financial experts, mathematicians and legal experts. Cryptographic schemes are the backbone to the protocols used to securing web browsing today. They ensure message confidentiality. The way we prove our credentials online using cryptographic techniques has led to an explosion in online business and banking. Cryptography is the invisible shield which guards our online transactions.

This article sketches some instances where cryptography is used to securing online transactions. We also touch upon some new topics, like privacy preserving computation and quantum cryptography, which are coming in vogue. Thus, cryptography presents numerous avenues for taking transactions to digital domain and in a secure fashion too.
\end{abstract}

Keywords: Cyptography; Communication; Banking; Digital transactions

\section{Introduction}

Shafi Goldwasser and Silvio Micali were awarded the ACM Turing Award in the year 2012. Their work (Goldwasser and Micali, 1984) had laid the foundations for security notions for today's browsers. They were lauded for their 'innovative approaches to ensuring security in digital age'. Whitfield Diffie and Martin E. Hellman were the recipients of ACM Turing Award for the year 2015. They received the award for their 'critical contributions to modern cryptography'. The Diffie-Hellman protocol (Diffie and Hellman, 1976) was recognized as protecting 'daily Internet communications and trillions of dollars in financial transactions' and their work was lauded as 'fundamental for our industry'. The pioneers' work was recognized as having made 'encryption technologies accessible to individuals and companies'. Their work is behind the most commonly encountered 'https' method of browsing the Internet, for example. This article discusses some of the fundamental cryptographic concepts and schemes which are pivotal for secure digital communications.

Human society has always aspired to hide important communications from prying eyes. Most of the techniques used historically were not robust enough to withstand attacks by clever adversaries. Cryptography (Katz and Lindell, 2014) is a collection of techniques which provides mechanisms for securing (digital) communications by ensuring confidentiality of the message and authenticity of entities. The communication will not be compromised even in the presence of a reasonably powerful adversary. Ideas from computer science, electronics, mathematics and physics are used to design such robust schemes. In a peer-to-peer communication model, cryptography assumes that the channel

*Authorfor Correspondence: E-mail: balu@imsc.res.in; prem@setsindia.net

${ }^{\#}$ Lecture delivered in the Mathematics and Society Symposia during the INSA Anniversary General Meeting, 2019 at CSIR-NIO, Goa 
connecting the peers can be listened to. The target is to give mechanisms for securing this communication. If the two peers have some secret known only to them, many mechanisms exist to ensure message confidentiality. This body of ideas is called symmetric key cryptography. But most often than not, the two peers are complete strangers to each other. The work of Diffie and Hellman (1976) laid foundation for public key cryptography, where two related keys, called encryption key and decryption key, are involved. The design is such that computing the secret key from the public key should be infeasible. This idea has revolutionized the way parties communicate over the internet. Tasks which involved human to human negotiations moved to purely digital domain. We discuss some current topics and future ones which illustrate relevance of cryptography to a digitally connected society. The second category includes those ideas which have not yet been commercialized on a large scale, but are very promising. While TLS and Whatsapp security fall in the first category, secure data analytics and quantum cryptography fall in the latter category.

The Transport Layer Security (TLS) protocol (Rescorla, 2018) is probably the best example for illustrating the importance of cryptography. The TLS protocol is used in the 'secure mode' of Internet browsing. Email communications, banking transactions and e-commerce are some of the applications of TLS protocol. This protocol first negotiates a common key between the two parties after authenticating them and then encrypts all communications using this key. Digital signature, a component used for authentication in the TLS protocol, has transformed the way we prove our credentials. Some of the applications of this technology include signing documents digitally and verifying our online tax returns. Zero Knowledge Proof (Goldwasser, Micali and Rackoff, 1989) is a future technology which could address the short comings of today's authentication mechanisms. This can be used to overcome the fear of password/credential theft. Using such a proof the owner can prove that he knows the password without actually revealing it. The other party is not only convinced of the credentials, but also learns nothing about the password/credentials. Specialized cryptographic schemes are used to secure messaging applications like Whatsapp.
Owning and processing volumes of data is the basis for offering "analytics as a service". Examples of data include network logs and hospital health records. Machine learning algorithms can be used to analyse such data and provide insights for future planning. Cryptographic techniques can be used in conjunction with some of these machines learning algorithm, thereby securing the process (Graepel, Lauter, and Naehrig, 2013). Another threat to such analysis is profiling of individuals/entities using the data. This arises when the custodian of the data disseminates some statistics based on the data. It is desirable to have mechanisms for data analysis which guarantee privacy. In other words, an adversary will not be able to identify the individual by looking at the output of such an analysis. Some research has been done on quantifying and defining privacy offered by data analysis algorithms. A sufficiently comprehensive definition is differential privacy. Differential Privacy (Nissim et al., 2017) is a set of criteria which data analysis algorithms are expected to satisfy, in order to ensure privacy of participating individuals. Techniques exist for making certain data analysis algorithms differentially private.

Quantum computing (Feynman, 1982) presents an alternative model for computing. The advantage is that problems, like factoring, which are hard to solve using classical computers, would become easy on an operational quantum computer (Shor, 1994). Thus, a quantum-enabled adversary would be able to read all the messages encrypted using RSA cryptosystem. On the other hand, Quantum Key Distribution (Bennett and Brassard, 1984), provides a mechanism for the key agreement. Assured by physical laws, this method achieves the best possible security. Thus, huge investments are being made by corporates and government for implementing this technology on to a portable device. Such QKD devices would be used to secure high value communications. In the meantime, post quantum cryptography (PQC) techniques are foreseen to replace quantum vulnerable ones. Such PQC systems would use classical systems and techniques, but would be resistant to quantum attacks. The PQC Competition, being conducted by NIST, is in its third round (Alagic et al., 2020). The final portfolio of schemes are expected to be the backbone of future Internet protocols like TLS.

In Section 2, we outline some basic notions of 
cryptography. In Section 3, we discuss TLS and its components. This is an internet protocol used for achieving confidentiality and authentication over net. In Section 4, we discuss some ideas which can be used for ensuring privacy and confidentiality of data analysis algorithms. In Section 5 we discuss emerging opportunities of quantum technology to securing communications. We present some concluding remarks in Section 6.

\section{Informal Description of Cryptography Notions}

We discuss some basic ideas which are relevant to this article. More details can be learnt from Stinson (Stinson, 1995) and (Katz and Lindell, 2014). Cryptology is the study and practice of techniques for secure communications in the presence of adversary. It is derived from Greek words "kryptos" meaning hidden or secret and "logia" meaning study. Cryptology comprises of:

- Cryptography-Design of secure systems

- Cryptanalysis -Analyzing or breaking such systems

It is very ancient and has found mention in various scriptures. It is very important in securing defence, banking and government transactions. In modern history, the encrypting device Enigma ${ }^{1}$ has played an important role in deciding the course of the Second World War. Invented by the German engineer Arthur Scherbius, it was used by German army during the Second World War. The device was first broken by Polish Cipher Bureau, as a result of the efforts of Rejewsky, Rozycki and Zygalski. It was broken mainly due to human errors and operational/procedural flaws, though there were some weaknesses present in the system. This played a major role in the Allies winning the war.

In 1883 Auguste Kerckhoffs wrote two journal articles on La Cryptographie Militaire, in which he stated six design principles laid down for military ciphers. In modern cryptologic parlance, it is stated as: "A cryptosystem should be secure even if everything about the system, except the key, is public knowledge."

\subsection{Goals of Cryptography}

Using cryptology, we hope to achieve

\footnotetext{
${ }^{1}$ http://en.wikipedia.org/wiki/Cryptanalysis_of_the_Enigma
}

Confidentiality: No third party can read the data

Authentication: The sender knows that the receiver knows a particular private key

Identification: Binding between the private key and the individual

Trust: Third party certifies that a particular private key belongs to a particular individual

Integrity: Messages are not corrupted during transit

\subsection{Setting and Types}

The setting for cryptographyis as follows. We discuss message confidentiality. Sender encrypts message using a key and sends it through a public channel, which the receiver decrypts. The encryption and the decryption keys may not be the same.

1.1.1. Symmetric Key. In symmetric key cryptography the encryption and the decryption keys are essentially the same. Such algorithms assume that the two parties have the same key. Two broad class of primitives are used to provide data confidentiality:

(1) Stream Ciphers: Examples include Trivium (De Canni'ere and Preneel, 2008) and Grain (Hell et al., 2006)

(2) Block Ciphers: Examples include Advanced Encryption Standard (AES) (Daemen and Rijmen, 2000) and PRESENT (Bogdanov et al., 2007)

Hash Functions are used to provide integrity and authentication.

2.2.2. Public/Asymmetric Key. Limitations of Symmetric Key Systems are as follows:

(1) Key Distribution

(2) Storage and management of large number of secret keys

(3) Cannot be applied to open systems, where parties have previously not met

A Key Distribution Centre (KDC) can be used for overcoming the above limitations. But such a centre is not practical in the case of open systems. Moreover, attack on the KDC breaks the system. Public key systems are used to overcome these short comings. 
In public key encryption schemes, the sender uses the receiver's public key to encrypt the message. The receiver, who alone knows the corresponding secret key, decrypts the cipher using this secret information. We describe the 'textbook' version of El Gamal encryption scheme below:

Setup: Let $\mathcal{F}_{p}^{*}=\langle\alpha\rangle$. Then $a$ is chosen at random and set $\beta=(\alpha)^{a}(\bmod p)$.

Set $P=(p, \alpha, \beta)$ and $S=a$.

$\operatorname{Encrypt}(\mathbf{x})$ : Choose $k$ at random. Set $e(x, P)=y=$ $\left(\alpha^{k}(\bmod p), x \beta^{k}(\bmod p)\right)$.

$\operatorname{Decrypt}(\mathbf{y})$ : For $y=\left(y_{1}, y_{2}\right)$, define $d(y, S)=$ $y_{2}\left(y_{1}^{a}\right)^{-1}(\bmod p)$.

Under the assumption that computing discrete $\log ^{2}$ is hard over finite fields, the above scheme is secure.

\section{Achieving Secure Communication over the Internet}

We discuss some cryptographic principles used in everyday internet protocols. These protocols ensure confidentiality, authenticity and integrity of the message. Authenticating users of services like email is also essential for secure communication. This section deals with four such topics, namely importance of the Transport Layer Protocol (TLS), usefulness of digital signatures, usage of zero knowledge proofs for authentication and cryptography used in Whatsapp. TLS protocol facilitates secure connection to remote servers. Use cases include netbanking and secure email. We discuss some crypto building blocks of this protocol. Digital signatures have revolutionized the way we prove credentials. It is also a component of the TLS protocol. Zero knowledge proofs provide a mechanism for authentication, where the credentials are proved without actually revealing the secret information. The Signal protocol is used for securing Whatsapp chats and media.

${ }^{2}$ Given a cyclic group $G=\langle g\rangle$ and $h \in G$, the discrete logarithm problem asks for $t$ such that $h=g^{t}$.

\subsection{TLS and its Importance}

Most of the digital infrastructure is protected by Transport Layer Security (TLS) (Rescorla, 2018). It is a cryptographic protocol used for securing digital communications over a network. This protocol is used to secure a wide range of applications like email exchange, Virtual Private Network establishment, web browsing and internet telephony. The primary use of this protocol is to secure the communication between a server website and a client browser. Rigorous proof of security exist for TLS 1.3. This section discusses cryptographic components of this protocol. We also touch upon its various applications.

2.1.1. Informal Description of the Protocol. The setting is as follows. Two parties, client and a server, want to authenticate each other and establish a common key to be used in encryption. The client could be a user trying to log into a webserver, for example. The protocol has two parts, namely, handshake and record. The handshake is the part where authentication and key agreement take place. The common key is used for encryption in the record part. The client contacts the server and requests a secure channel. The server replies with possible cryptographic algorithms which the client can use for this purpose. The client chooses one and intimates the server. Now, the client wants an assurance that it is indeed talking to the correct server. This verification is done by the client when the server submits its digital certificate. More details on this topic will be discussed in Subsection 3.2. Now, the client and the server proceed to the key agreement phase.

It must be noted that the two parties do not have any common secret information to begin with. The key agreement is achieved using the Diffie-Hellman protocol described below. This mechanism allows the two parties A and B to arrive at a common key.

Require: A cyclic group $G$ which is generated by $g$, so that $G$ is completely described as powers of $g$.

Party A: This party chooses $a \in\{0, \ldots, n-1\}$ secretly, computes $g^{a}$ and send to Party B.

Party B: Similar action as above by this party too, where she chooses $b \in\{0, \ldots, n-1\}$ secretly, computes $g^{b}$ and sends to Party A.

Common Key: Party A takes $g^{b}$ received from Party 
B and computes $K=\left(g^{b}\right)^{a}=g^{a b}$, using its secret choice $a$. Party B computes the same key analogously.

Of course, we need cyclic groups where the DH problem (Problem 1) is hard. Certain subgroups of the multiplicative group of finite fields and elliptic curves are used for instantiating this scheme.

\section{Problem 1. Diffie-Hellman Problem}

Input: Cyclic group $G=\langle g\rangle$, and $g^{a}, g^{b}$

Output: Compute $g^{a b}$

Confidentiality is achieved using encryption. PRESENT (Daemen and Rijmen, 2000) or TRIVIUM (De Canni'ere and Preneel, 2008) can be used for this purpose. The session proceeds by using this common key for encrypting all communications using some symmetric key ciphers. Since the key is known only to the two parties, even if the communication is intercepted, it will not be intelligible. A formal proof of security of the entire protocol is more involved.

3.1.2. Use Cases. Numerous portals offering online services use TLS for secure communication. Online shopping portals and netbanking sites are typical examples. Many email service providers use TLS. This protocol can be used as the backbone for executing a secure video conferencing application. This is also useful for secure access of webpages, like income tax e-filing of tax returns. It is hard to imagine secure web without the TLS protocol.

\subsection{Digital Signatures and its Importance}

We have seen that encryption techniques can be used to ensure confidentiality of the message. An independent requirement in any digital communication is that of authentication. Servers, websites, individuals, emails and software are some examples of entities which need authentication. A related requirement is that of non-repudiation. It is also desired that a communication that has been tampered with en route can be identified (integrity check). These problems arise due to the fact that it is not difficult to intercept and modify digital communications. A malicious element may pretend as another entity and send improper communication. Authentication, integrity check and non-repudiation are achieved using digital signatures. Entities which need authentication include email messages, software codes, documents and servers. We illustrate this notion with some motivating examples.

(1) Suppose a server wants to push a software patch to a genuine client. How can the client verify that the patch is indeed a valid one from the server and not a piece of malicious code pushed by some miscreant?

(2) An email user has received an email from an acquaintance. How can she be assured that the missive is indeed genuine and from that acquaintance?

\subsubsection{Informal Description of Digital Signatures.}

Informally, what is desired is a binding between the sender and the payload. In the first case, the binding is expected between the server and the patch. And in the case of email, the binding between the missive and the sender is expected. Such a requirement is satisfied using a digital signature.

We describe this notion. A digital signature, defined in cryptography, has three algorithms. The first one, called Key Gen, outputs a pair of keys called the signing key and the verifying key. The signing key, as the name suggests, is used to sign a message. A string called signature is generated from the message using the signing key. This is implemented using the Sign module. Needless to say, this signing key is to be kept secret by the user. When the message is received together with the signature, Verify implements a mechanism to check that the message is genuine. This uses the verification key of the sender. The verification key is a public information. Anyone with a message-signature pair from a particular sender can verify the genuinenesss using the sender's verification key. We formally define the notion below:

Definition 1. A digital signature scheme is a triple of $P P T^{3}$ algorithms (KeyGen, Sign, Verify) where

- KeyGen outputs the public key and secret key of a user for a security parameter

- $\quad$ Sign takes as input a message and the secret key of the signer and outputs a signature

${ }^{3}$ Probabilistic Polynomial Time 
- Verify takes the message, signature and the public key of the signer to output either accept or reject

The signature scheme needs to be correct, in the sense that upon input of correct signature of the message, the verify should always accept and wrong signature should be rejected with high probability.

Let us discuss the security of such a notion. The goal of the miscreant (called adversary) is to impersonate a particular user and tamper with the user's communication. In other words, the adversary tries to come up with a valid message-signature pair and send it, in such a way that it looks like a genuine missive from that user. But the signing key of the user is secret and not known to the adversary. The adversary may attempt to perform a forgery, that is, obtain a valid message signature pair for a particular user. The signature scheme is said to be secure if it is unforgeable. Some additional powers may be given to the adversary for getting stronger security guarantees. The designer of the digital signature would argue that if an adversary could get a forgery, then, that adversary would be able to solve a mathematically hard problem. We describe the existential unforgeability under chosen message attack game in Definition 2.

Definition 2. The signature experiment Sig forge $_{\mathcal{A}, \pi}(n)$

(1) KeyGen $\left(1^{n}\right)$ outputs (pk, sk).

(2) The adversary $\mathcal{A}$ is given pk and access to the signing oracle. Let $\mathcal{Q}$ be the set of queries which the adversary has made. The adversary then outputs $(m, \sigma)$.

(3) The adversary succeeds if Verify $(p k, m, \sigma)=$ 1 and $m \notin \mathcal{Q}$.

A signature scheme $\pi=($ KeyGen, Sign, Verify) is said to be existentially unforgeable under an adaptive chosen-message attack (EUFCMA) or just secure if for all probabilistic polytime adversaries $\mathcal{A}$, there is a negligible function negl such that

$$
P\left[\operatorname{Sig}-\operatorname{forge}_{\mathcal{A}, \pi}(n)=1\right] \leq \operatorname{negl}(n) .
$$

3.2.2. An Example. We describe the RSA signature scheme below. We do not dwell on the implementation specifics like key sizes.

KeyGen: Choose two large primes $p$ and $q$ and calculate $n=p q$. Choose the verification key $e$ such that it is not a factor of $(p-1)(q-1)$. Compute the (unique) signing key $d$ such that $d=e^{-1}(\bmod (p-1)$ $(q-1))$. The 'modulus' is considered to be public information.

Sign: Let $M$ be the message that needs to be signed by the signer. The signer computes $\sigma=M^{d}(\bmod n)$.

Verify: For the message-signature pair $(M, \sigma)$, the verifier computes $T=\sigma^{e}(\bmod n)$. The verifier accepts if $T=M$.

Correctness follows easily from Euler's theorem ${ }^{4}$. The security of this scheme is related to the factoring problem (Problem 2). This problem is considered to be computationally hard.

\section{Problem 2. Factoring}

Input: Given a positive integer $N$ satisfying $N=p$ $\cdot q$, for large primes $p$ and $q$.

Output: One factor of $N$.

3.2.3. Practical Uses of Signatures. The Public Key Infrastructure (PKI) (Chokhani et al., 2003) sets down policies, roles of entities and software/ hardware components for creation, management and revocation of digital certificates. As we have seen in the above discussion, signature binds the entity and the key. A certifying authority (CA) verifies this binding before issuing the digital certificate. Registration, verification, issuance and revocation are carried out by the $\mathrm{CA}$ in accordance with a set policy.

Digital signatures are widely used for various transactions in India. Guidelines for usage have been set by the Information Technology Act (amended in $2008)^{5}$. An entity called $e M u d r a^{6}$ is a Certifying Authority which issues digital signatures to customers. Six kinds of certificates are issued, viz., signature, encryption, device/system, SSL server, code signing

\footnotetext{
${ }^{4}$ Recall that the totient function $\varphi(n)$ counts number of positive integers less than $n$ and relatively prime to $n$. Euler's theorem asserts that $a^{\varphi(n)} a \equiv 1(\bmod n)$, when $a$ and $n$ are relatively prime. ${ }^{5} \mathrm{https} / / /$ www.meity.gov.in/content/information-technology-act2000

${ }^{6}$ https://www.e-mudhra.com/
} 
and document signing. Digital signatures are used by entities for communications with departments like income tax department, GST authority, provident fund, banking, e-procurement and foreign trade.

\subsection{Zero Knowledge Proofs}

We discuss a not-too-popular mechanism for proving credentials in a digital communication. There is a persistent threat that passwords stored in systems can be stolen. Such events have caused serious damage in terms of financial, personal and material loss. Introduced by (Goldwasser, Micali and Rackoff 1989), zero knowledge proofs can prevent such mishaps, while still allowing authentication (and many other functionalities). The client can 'prove' to the server that it knows its password without actually revealing any other information about the password.

3.3.1. Informal Description. We describe the notion of zero knowledge proof system in a non-technical fashion. The prover $P$ has a secret information. $\mathrm{He}$ wants to convince the verifier $V$ that he has this information. The secret information could be a secret key or a password. The system incorporates adversarial behavior by modelling dishonest prover and verifier. A dishonest prover is one who does not have this secret key but is trying to convince a honest verifier that he knows it. A zero knowledge proof system satisfies three properties:

Completeness: if the statement is true a honest prover will always be able to convince a honest verifier about the truth of the statement.

Soundness: if the statement is false, no dishonest prover will be able to convince a honest verifier, except with a small probability.

Zero Knowledge: if the statement is true, no verifier learns any other information except that the statement is true.

Let us see a practical example of such a protocol between a prover $P$ and a verifier $V$. The prover wants to prove the knowledge of a discrete $\log ^{7}$. That is, on a cyclic group $G=\langle g\rangle$, for an element $h$, the prover $P$ wants to prove that she knows $a$ such that $h=g^{a}$. The scheme works as follows. The group description $G=\langle g\rangle$ is known to both $\mathrm{P}$ and $\mathrm{V}$. The prover $P$

\footnotetext{
${ }^{7}$ The problem of computing discrete log is hard on certain groups.
} Such groups are chosen. chooses $r$ at random, computes $g^{r}$ and sends to $\mathrm{V}$. The verifier $\mathrm{V}$ asks $\mathrm{P}$ to reveal one of the two values, viz., $r$ or $a+r$. Since $r$ is random, when revealing $a+$ $r$ prover $\mathrm{P}$ is giving only a random value.

3.3.2. Use Cases. Potentially, zero knowledge proofs can be used wherever some form of authentication mechanism is needed. These are not very popular now because of the overheads involved over existing authentication mechanisms. A password based login used in netbanking, social media site or email account can be replaced with a zero knowledge based authentication mechanism. Zero knowledge proofs for passwords have been constructed. This cryptographic idea is poised to revolutionize digital payment industry. It is already being used in cryptocurrencies ${ }^{8}$.

\subsection{Cryptology in Whatsapp}

Most individuals use some social media platform or messaging applications. Facebook, Twitter and Whatsapp are examples of such applications. Sensitive information, photographs and other documents are shared over such platforms. Thus it becomes vital for the user to ensure that the custody of such information is in safe hands. Many of these applications use proprietary protocols for ensuring security. Needless to mention, cryptography forms an important part of these protocols. We take the example of Whatsapp to demonstrate this fact.

The Signal protocol, based on Double Ratchet, designed by Open Whisper Systems is used by Whatsapp for providing end-to-end security ${ }^{9}$. It is claimed that no one other than the intended parties, including Whatsapp, can read messages. Group chats, media and calls too are allegedly end-to-end encrypted. The protocol is also claimed to offer perfect forward secrecy. That is, even if the phone is compromised and Whatsapp keys revealed, older messages cannot deciphered. This is ensured by changing keys from session to session. The core building blocks of key derivation is the elliptic curve Diffie-Hellman protocol on Curve 25519 (Bernstein, 2006). AES 256 and HMAC-SHA 256 are used for

\footnotetext{
${ }^{8}$ https://www.technologyreview.com/2017/11/09/3857/ a-mind-bending-cryptographic-trick-promises-to-takeblockchains-mainstream/

${ }^{9}$ https://www.whatsapp.com/security/WhatsApp-SecurityWhitepaper.pdf
} 
ensuring message confidentiality and authentication.

The security of the alleged protocol is now an object of intense study (Dowling and Hale, 2020; Chase, Perrin and Zaverucha, 2019). The wide spread use of Whatsapp has led to intense scrutiny of the said protocol. However, undeniably, cryptography is the crux for ensuring security of individual chats over such media.

\section{Privacy Preserving Computation}

Many corporate and government agencies, collect, use and share data which can be used to directly identify participating individuals. Improper dissemination of such data can be harmful to the subject, causing financial and social loss. It can also result in penalization of the custodian of data. The custodian of the data may also be mandated to publish summary statistics of the data. In this section we describe the notion of differential privacy (DP) and its relevance to privacy preserving statistical computation. Then we discuss some cryptographic techniques like homomorphic encryption and multiparty computation. These are useful for operating on encrypted data.

\subsection{Differential Privacy}

It is widely believed that privacy is a property of the analysis and not of its outcome. With foundational work by (Dwork, 2006) differential privacy overcomes the limitations of anonymisation techniques. Differential Privacy is a set of criteria which statistical analysis tools are desired to satisfy. These criteria are set in such away that they provide a mathematically provable guarantee of privacy protection against a wide range of privacy attacks. The attacks include re-identification, record linkage and differencing. The goal, here, is not to stop the adversary from gaining unauthorized access to the system. Rather, the goal is to allow access to database but stop leakage of individual information after successive queries. Same inference about any individual's private information will be drawn by the person observing the result, whether or not the individual's private information was included in the input of the analysis.

Differential Privacy techniques can be used in conjunction with certain machine learning algorithms.
The benefits of incorporating such techniques are as follows. Privacy guarantee against successive data releases is ensured. Protection against a wide range of potential privacy attacks, including some of those which were not envisaged during the design phase, is promised. Correctly implemented, public access to data and summaries can be granted, without privacy concerns. It may be noted that these techniques are transparent and do not affect the accuracy of the results (Abadi et al., 2016). Thus, the training phase of a ML algorithm for example, can incorporate tools for providing privacy. Such techniques have also been used in certain machine learning algorithms for clustering and classification.

4.1.1. Informal Description. Differential privacy ensuring techniques are applicable for statistical databases, where the target is to ensure privacy of individual-specific information and the custodian of the data is trusted (Nissim et al., 2017). The setting is as follows:

(1) There is one database and the target is to release summary statistics without allowing access to individual records

(2) Attacker can access that database and must learn aggregate statistics, but not any new information about individuals in database

(3) What the attacker has learnt must not identify an individual

Differential privacy assures that same result would be obtained whether or not a particular user's data was or was not used in the statistical computation. This condition should hold for all users. This is achieved by adding random noise to data. We give the formal definition (Definition 3) just for the sake of completeness. The reader may refer to (Dwork et al., 2006) for more details.

Definition 3. Differential Privacy A randomized algorithm $\mathcal{M}$ with domain $\mathbb{N}|\chi|$ is said to be $(\varepsilon, \delta)$ differentially private if for all $\mathcal{S} \subseteq$ Range $(\mathcal{M})$ and for all $x, y \in \mathbb{N}|x|$ such that $\|x-y\|_{1}<1$ :

$$
P[\mathcal{M}(x) \in \mathcal{S}] \leq \exp (\varepsilon) P[\mathcal{M}(y) \in \mathcal{S}]+\delta,
$$

where the probability is over random coins of $\mathcal{M}$. The notation $\|x-y\|_{1}<1$ stands for the statement that databases $x$ and $y$ differ in utmost one record. 
4.1.2. Use Cases. Differential privacy has proved to be useful for data scientists, researchers, policy makers and corporates. The U.S. Census Bureau has developed a 'On The Map' application as part of the Longitudinal Employer-Household Dynamics programme. DP has been used for protecting the block-level residential population data. Apple collects user preferences and this data was used, for example, to discover most popular emojis and memory usage in a particular application. DP was used to protect the user identity. Microsoft has developed 'PrivTree' for providing location-based services to users. This application blurs the geo-location information of the individual using DP techniques. Google has developed 'Randomized Aggregatable Privacy-Preserving Ordinal Response (RAPPOR)' for crowd sourcing end-user client software statistics anonymously.

\subsection{Cryptography for Machine Learning}

Keeping the data and model safe during the ML process is a natural security goal. Cryptographic promises can propel people towards using 'Machine Learning as a Service'. Cryptography and machine learning have developed together through mutual interactions and contribution. The Learning Parity with Noise (LPN) problem and Learning With Errors (LWE) problem, having origins in ML, have resulted in building of secure cryptosystems. These assume importance because these underlying hard problems are not solvable even by a quantum computer. Many cryptographic primitives have been used in ML systems for achieving the above goal (Graepel, Lauter, and Naehrig, 2013).

Homomorphic encryption allows operating on encrypted data. Machine learning algorithms like neural networks have been implemented to work on encrypted data. Highly sensitive data like genomic data has been operated upon after encryption using such techniques. Secret sharing is a cryptographic mechanism of protecting a highly sensitive piece of information, which cannot been trusted with one person. The participants are given shares in such away that only authorized sets of participants can come together to recover the information. Any unauthorised set of users can get no information about the grand secret. Such technique shave been used to de-centralize machine learning, both the training and model building phases. Techniques like Multi-Party
Computation and garbled circuits have also been applied in machine learning.

\section{Impact of Quantum Principles on Crypto- graphy}

Quantum computing uses techniques from quantum physics, maths and computer science to build a different model for computation. Works of Benioff (Benioff, 1980) and Feynman (Feynman, 1982) laid the foundations of such a model. Quantum Turing machine and quantum circuit are some well-known models for quantum computation. These use principles like entanglement and superposition. Shor's algorithm (Shor, 1994) proved that quantum computers can perform factoring and discrete log computation in polynomial time. Classical computers take sub exponential time to solve this problem (Pomerance, 1996) even today. This directly renders classically secure RSA and DH cryptosystems insecure in the presence of a quantum adversary. Other mathematical hard problems like finding shortest vector on lattices and solving a system of quadratic equations in many variables over a finite field do not yet have any polytime quantum algorithms. Quantum algorithms providing exponential speed-up. For breaking symmetric ciphers like AES do not exist yet. Doubling the secret key/digest size is generally sufficient. Thus, the term post-quantum cryptography is used only in the context of public key cryptography. Refer (de Wolf, 2017) for a physicist's perspective.

On the other hand, quantum particles (qubits) can be transmitted from one point to other and measured. Such a transmission cannot be tamperedwith by adversaries. This forms the basis of a mechanism for key agreement called quantum key distribution (QKD) (Bennett and Brassard, 1984). The QKD algorithm behaves like a one-time pad and offers 'information theoretic security'. Such a claim is based on physical properties of quantum particles.

Thus, on the one hand, it is imperative to find alternatives for RSA and DH cryptosystems. A quantum-enabled adversary would break these in polynomial time. On the other hand, QKD offers a viable mechanism for key agreement, offering best possible security. This section describes these two approaches:

(1) Usage of quantum key distribution for arriving 
at the common secret key between two parties and then using symmetric ciphers (on classical computers) and

(2) Replacing RSA/DH based public key systems with other quantum resilient ones.

\subsection{Quantum Key Distribution}

Knowledge of a common secret key is a precursor to any secure peer-to-peer communication. Quantum Key Distribution (QKD) (Bennett and Brassard, 1984) is a mechanism by which such a common key can be agreed upon. Laws of quantum mechanics assure that such a key distribution mechanism remains private to the two peers. This common secret key is used for further encryption/ authentication requirements. QKD is usually described as a mechanism for getting common secret key for use in a one-time pad.

We describe the QKD algorithm briefly. To prevent man-in-the middle attack an authentication, based on pre-shared common key, is first performed. Photons (with random chosen polarization) are transmitted by the sender to the receiver. They agree upon four different polarization states for encoding classical bits, which is done over a classical channel. The receiver chooses random filter orientations and records the outcome. After the transmission is complete, the receivers ends the orientations of the filters to the sender. The sender responds with a list of compatible orientations. In 'sifting phase' the bits in non-compatible orientation positions are discarded. Two parties then reveal some bits to check for errors. This part of the common key is discarded. An error correcting code is applied on the common key to take care of errors introduced by eavesdropper, channel imperfections and environmental perturbations. Then privacy amplification is performed to minimize information of the eavesdropper. A simple sumwithout-carry of two consecutive bits is performed. A small portion of the common key is reserved for next round of authentication.

There are commercially available QKD boxes which allow $100 \mathrm{Gbps}$ data-in motion encryption. These use QRNG for basis/polarization choice. Use cases include telecom service provider, financial services, banks, cloud, health care, and government. These use dedicated hardware for performing AES encryption. QKD has been successfully deployed using fibre optic cables and free space over $100 \mathrm{~km}$ range. Key rate of a few Mbps speed has been achieved. National Critical Infrastructure Protection seems to be the driving force for huge investments by governments.

5.1.1. Challenges. QKD is no longer seen as a technology confined to labs, but as a evolving solution to key management problem of defence agencies. Rapid deployment of this technology is hampered by highly specialized attacks on such systems and also engineering considerations (Diamanti et al., 2016). Research is on to construct a hardware resilient to side channel attacks (attacks which measure parameters like electromagemtic radiation emitted by the operational device to discern some secret information). Secret key needs to be generated at high bitrate for high volume encryption. Attenuation of light transmitted over fibres at large distances seems to put a limit to fibre-based QKD.

\subsection{Post-Quantum Cryptography}

The RSA and DH cryptosystems form the core of important internet protocols like TLS. These cryptosystems are used as KEM, key agreement and signatures. Planning for the future, quantum resilient TLS protocol is imperative due to Shor's quantum algorithm. Rather than discussing the whole protocol, we focus only the public key cryptography components of the protocol and their quantum resilience. We would give examples and descriptions only for digital signatures. We will mention KEM and key agreement in the passing.

Quantum computers do not affect the security of symmetric key systems much. Thus, post-quantum cryptography is the study of public key cryptosystems in the following setting:

(1) Only classical devices like laptops, routers and servers would be used for performing cryptographic operations like signing and verification. Devices operating on quantum principles will not be used,

(2) The adversary is assumed to be quantumenabled. He can use quantum principles to attack cryptosystems and

(3) Primitives like Key Encapsulation Mechanism, key agreement and signatures are of interest. 
Let us discuss fundamental steps involved in building quantum resilient cryptosystems. We concentrate on signatures for illustrative purposes. The first requirement is a hard problem which is resilient to quantum attacks. Certain computational problems on lattices, codes and polynomial algebras are known to be quantum resilient and also hard classically. We describe the Multivariate Quadratic (MQ) problem in Problem 3:

\section{Problem 3. $M Q$ Problem}

Input: A system of multivariate quadratic polynomials over a finite field $\mathbb{F}_{q}$ is given:

$$
f_{1}\left(x_{1}, \ldots, x_{n}\right), \ldots, f_{m}\left(x_{1}, \ldots, x_{n}\right)
$$

Output: $A$ solution $x_{1}=\alpha_{1}, \ldots, x_{n}=\alpha_{n}$ satisfying this system

The MQ problem is known to be NP-complete. The best quantum attack on this problem gives only a square root speed up (Bernstein and Yang, 2018). Thus, this problem can be a suitable candidate for building a cryptosystem. The Unbalanced Oil Vinegar (UOV) scheme (Kipnis, Patarin and Goubin, 1999) and Rainbow (Ding and Schmidt, 2005) are based on this problem. We describe the rainbow signature scheme.

Parameters: Finite Field $\mathbb{F}_{q}$; integers $0<v_{1}<\ldots v_{u}$ $<v_{u}+1=n$; index sets $V_{i}=\left\{1, \ldots, v_{i}\right\}, O_{i}=\left\{v_{i}+1\right.$, $\left.\ldots, v_{i}+1\right\}(i=1, \ldots, u)$, such that each $k \in\left\{v_{1}+1\right.$, ..., $n\}$ is contained in exactly one $O_{i}$; set $o_{i}=\left|O_{i}\right|$; number of equations $m=n-v_{1}$; number of variables $n$.

Key Generation: The secret key consists of two invertible affine maps $\cdot m \quad \cdot n \quad$ and the central quadratic map $\quad \cdot n \quad$ consisting of $m$ multivariate polynomials $f^{\left(v_{1}+1\right)}, \ldots, f^{(n)}$. The polynomials are of the form

$$
\begin{aligned}
& f^{(k)}\left(x_{1}, \ldots, x_{n}\right)=\sum \alpha_{i j}^{(k)} x_{i} \\
& +\sum \beta_{i j}^{(k)} x_{i} x_{j}+\sum \gamma_{i}^{(k)} x_{i}+\delta^{(}
\end{aligned}
$$

where $l \in\{1, \ldots, u\}$ is the only integer such that $k \in$ $O_{l}$. The coefficients $\alpha_{i j}, \beta_{i j}, \gamma_{i}, \delta_{i}$ all come the base finite field. The size of the private key is

$$
m(m+1)+n(n+1)+\sum^{u} \frac{v_{i}\left(v_{i}+1\right)}{-}+v_{i} o_{i}+v_{i}+o_{i}-
$$

field elements.

Public Key: The public key is the composite map

$$
P=\mathcal{S}_{\circ} \mathcal{F}_{\circ} \mathcal{T}
$$

consisting of $m$ quadratic polynomials. The size of the public key is

$$
m
$$

base field elements.

Sign: Let $d$ be the message to be signed. First compute $h=\mathcal{H}(d)$, where $\mathcal{H}:\{0,1\}^{*} \rightarrow \quad$ is a hash function. Then compute $\cdots \sigma^{-1} x-\quad$ Then compute $y=\mathcal{F}^{-1}(x)$. The signature $z=\mathcal{T}^{-1}(y)$ is then computed. The central map is inverted by assigning random values to vinegar variables and then Selving the Unear system.

Verify: Let $d$ be the message to be signed. First compute $h=\mathcal{H}(d)$. Then

$$
\text { Compute } h^{\prime}=\mathcal{P}(z) \text {. If } h=h^{\prime} \text { accept. }
$$

The second requirement is a formal model of security, which incorporates a quantum adversary. Whereever applicable, the quantum adversary should be allowed to exploit quantum principles. For example, a security proof against 'Existential Unforgeability Under Chosen Message Attack' in the Quantum Random Oracle Model (QROM) is desired for a digital signature.

5.2.1. Challenges. Rainbow signature, providing smallest target level of security, has public key size of $149 \mathrm{kB}$, secret key size of $93 \mathrm{kB}$ and signature size of about 400 bits. Compared to RSA these sizes are much higher. The signing and verification time is higher too in the case of Rainbow. The challenge now is to optimize parameter sizes and execution times of these new schemes, so that they become drop-in replacements for older schemes. 
The process for standardization of quantum resilient public key schemes in being done by NIST $^{10}$. KEM, PKE, key agreement and signatures are being considered. A few different algorithms are expected to be standardized. Security will be measured in terms of number of operations required to break AES128 / SHA256 / AES192 / SHA384 / AES256 for levels 1 to 5 , respectively. Performance will be measured on standard platforms. Other properties like resistance to side channel attacks, misuse resistance, must be drop-in replacements and perfect forward secrecy are expected of the candidates.

Four KEM/key agreement and three signatures have advanced into third round of the competition (Alagic et al., 2020). Once this competition is complete, the process of incorporating these schemes in protocols like TLS will be taken up. Such schemes will also be standardized for use in Trusted Platform Module (TPM) and Hardware Security Module (HSM).

\section{Concluding Remarks}

We outlined some applications used by us everyday that use cryptography. Transport Layer Security and

${ }^{10} \mathrm{https} / / / \mathrm{csrc}$. nist.gov/projects/post-quantum-cryptography

\section{References}

Alagic Gorjan, Alperin-Sheriff Jacob, Apon Daniel, Cooper David, Dang Quynh, Kelsey John, Liu Yi-Kai, Miller Carl, Moody Dustin, Peralta Rene and Perlner Ray (2020) Robinson Angela and Smith-Tone Daniel (2020) Status report on the second round of the nist post-quantum cryptography standardization process NISTIR 8309

Abadi Martin, Chu Andy, Good fellow Ian, McMahan H. Brendan, Mironov Ilya, Talwar Kunal and Zhang and Li (2016) Deep learning with differential privacy. In Proceedings of the 2016 ACM SIGSAC Conference on Computer and Communications Security, CCS '16, page 308-318, New York, NY, USA, Association for Computing Machinery

Bennett C H and Brassard G (1984) Quantum cryptography: Public key distribution and coin tossing. In Proceedings of IEEE International Conference on Computers, Systems, and Signal Processing, page 175

Benioff Paul (1980) The computer as a physical system: A digital signatures are safeguarding the way we do online banking transactions and purchase items using e-commerce sites. We presented the crypto techniques used in transport layer security protocol (TLS). We discussed the basics of key agreement and digital signatures with motivating examples. We also outlined how quantum technology is poised to revolutionize tomorrow's internet. We outlined one technique where quantum principles are directly used to achieve secure key management, viz., quantum key distribution. Post-quantum cryptography was described as a library of classical schemes, but which are quantum resilient. Secure and privacy preserving computation is another opportunity for cryptologists to come up with novel applications. Many ideas are emerging for operating on encrypted data effectively. Privacy preserving machine learning is gaining importance for its vast business opportunity.

It can be said confidently that cryptography will continue to play vital role in securing our online transactions. New schemes constructed after thorough research would provide greater security and functionality, thus securing our digital transactions.

microscopic quantum mechanical hamiltonian model of computers as represented by turing machines Journal of Statistical Physics, 22 563-591

Bernstein Daniel J (2006) Curve25519: New diffiehellman speed records. In Moti Yung, Yevgeniy Dodis, Aggelos Kiayias and Tal Malkin, editors, Public Cryptography- PKC 2006, pages 207-228, Springer Berlin Heidelberg.

Bogdanov Andrey, Knudsen Lars R, Leander Gregor, Paar Christof, Poschmann Axel, Robshaw Matthew JB, Seurin Yannick and Vikkelsoe Charlotte (2007) Present: An ultralightweight block cipher. In International workshop on cryptographic hardware and embedded systems, pp. 450466. Springer

Bernstein Daniel J and Yang Bo-Yin (2018) Asymptotically faster quantum algorithms to solve multivariate quadratic equations. In Tanja Lange and Rainer Steinwandt, editors, Post-Quantum Cryptography - 9th International Conference, PQCrypto 2018, Fort Lauderdale, FL, USA, April 9-11, Proceedings, volume 10786 of Lecture Notes 
in Computer Science, pp. 487-506. Springer

Chokhani S, Ford W, Sabett R, Merrill C and Wu S (2003) Rfc3647: Internet x.509 public key infrastructure certificate policy and certification practices framework

Chase Melissa, Perrin Trevor and Zaverucha Greg (2019) The signal private group system and anonymous credentials supporting efficient verifiable encryption. Cryptology ePrint Archive, Report 2019/1416, https://eprint.iacr.org/ 2019/1416

De Canni 'ere Christophe and Preneel Bart (2008) Trivium, pp. 244-266. Springer Berlin, Heidelberg

Diffie W and M Hellman (1976) New directions in cryptography IEEE Transactions on Information Theory 22 644-654

Dowling Benjamin and Hale Britta (2020) There can be no compromise: The necessity of ratcheted authentication in secure messaging Cryptology ePrint Archive, Report 2020/ 541, https://eprint.iacr.org/2020/541

Diamanti Eleni, Lo Hoi-Kwong, Qi Bing and Yuan Zhiliang (2016) Practical challenges in quantum key distribution. npj Quantum Information, 2(1), Nov 2016

Dwork Cynthia, McSherry Frank, Nissim Kobbi and Smith Adam (2006) Calibrating noise to sensitivity in private data analysis. In Shai Halevi and Tal Rabin, editors, Theory of Cryptography pp. 265-284, Springer Berlin Heidelberg

Daemen Joan and Rijmen Vincent (2000) Rijndael for AES. In The Third Advanced Encryption Standard Candidate Conference, April 13-14, 2000, New York, New York, USA, pp. 343-348. National Institute of Standards and Technology

Ding Jintai and Schmidt Dieter (2005) Rainbow, a new multivariable polynomial signature scheme. In John Ioannidis, Angelos D. Keromytis, and Moti Yung, editors, Applied Cryptography and Network Security, Third International Conference, ACNS 2005, New York, NY, USA, June 7-10, Proceedings, volume 3531 of Lecture Notes in Computer Science, pp. 164-175

de Wolf Ronald (2017) The potential impact of quantum computers on society. CoRR, abs/1712.05380.

Feynman Richard P (1982) Simulating physics with computers. 21 467-488. Physics of computation, Part II (Dedham, Mass., 1981).
Graepel Thore; Lauter Kristin and Naehrig Michael (2013) Ml confidential: Machine learning on encrypted data. In Taekyoung Kwon, Mun-Kyu Lee, and Daesung Kwon, editors, Information Security and Cryptology - ICISC 2012, pp 1-21, Springer Berlin Heidelberg

Goldwasser Shafi and Micali Silvio (1984) Probabilistic encryption J Comput Syst Sci 28 270-299

Goldwasser Shafi, Micali Silvio and Charles Rackoff (1989) The knowledge complexity of interactive proof systems SIAM Journal on Computing 18 186-208

Hell Martin, Johansson Thomas, Maximov Alexander and Meier Willi (2006) A stream cipher proposal: Grain-128. In Proceedings 2006 IEEE International Symposium on Information Theory, ISIT 2006, The Westin Seattle, Seattle, Washington, USA, July 9-14, 2006, pp 1614= 1618. IEEE

Katz Jonathan and Lindell Yehuda (2014) Introduction to Modern Cryptography, Second Edition. Chapman and Hall/CRC, 2nd edition

Kipnis Aviad, Patarin Jacques and Goubin Louis (1999) Unbalanced oil and vinegar signature schemes. In Jacques Stern, editor, Advances in Cryptology EUROCRYPT'99, International Conference on the Theory and Application of Cryptographic Techniques, Prague, Czech Republic, May 2-6, 1999, Proceeding, volume 1592 of Lecture Notes in Computer Science, pp. 206-222. Springer

Nissim Kobbi, Steinke Thomas, Wood Alexandra, Altman Micah, Bembenek Aaron, Bun Mark, Gaboardi Marco, O’Brien David R and Vadhan Salil (2017) Differential privacy: A primer for a non-technical audience. In Privacy Law Scholars Conf, volume 3

Pomerance Carl (1996) A tale of two sieves, Notices Amer Math Soc 43 1473-1485

Rescorla Eric (2018) The Transport Layer Security (TLS) Protocol Version 1.3. RFC 8446, August

Shor P W (1994) Algorithms for quantum computation: discrete logarithms and factoring. In Proceedings 35th Annual Symposium on Foundations of Computer Science pp. 124134

Stinson Douglas R (1995) Cryptography - theory and practice. Discrete mathematics and its applications series, CRC Press. 